\title{
SPECIAL SOLUTIONS OF CERTAIN DIFFERENCE EQUATIONS.
}

\author{
By \\ F. JOHN \\ of Lexington, Ky., U.S. A.
}

Let $f^{\prime}(x)$ be a solution of the difference equation

$$
f(x+1)-f(x)=g(x)
$$

for $x>0$. $f(x)$ may be uniquely determined by prescribing its values arbitrarily for $0<x \leqq 1$. For certain functions $g(x)$ however the solution $f$ may also be characterized by simple properties, instead of prescribed values in an interval. A solution may e.g. be uniquely determined by its asymptotic behaviour for large $x$; this leads to the $\gg$ Hauptlösung» of the difference equation, as defined by N. E. Nörlund in his »Vorlesungen über Differenzenrechnung». In special instances solutions have also been characterized by local properties. Thus it has been proved by $\mathrm{H}$. Bohr, that for $g(x)=\log x$, all strongly convex solutions of (I) are of the form $f(x)=$ const. $+\log \Gamma(x) .^{1}$ Here a function is called »strongly convex», if

$$
f(\lambda x+(\mathrm{I}-\lambda) y) \leqq \lambda f(x)+(\mathrm{I}-\lambda) f(y) \text { for } 0 \leqq \lambda \leqq \mathrm{I},
$$

whereas "convexity" alone only implies, that

$$
f\left(\frac{x+y}{2}\right) \leqq \frac{f(x)+f(y)}{2} \cdot 2
$$

An analogous result has been derived recently by A. E. Mayer: ${ }^{3}$ The only convex solution of the functional equation $\mathrm{I} / f(x+\mathrm{I})=x f(x)$ is given by

$$
f(x)=\frac{\mathrm{I}}{\sqrt{2}}=\frac{\Gamma\left(\frac{x}{2}\right)}{\Gamma\left(\frac{x+\mathrm{I}}{2}\right)} .
$$

1 Cf. e. g. E. Artin: Einführung in die Theorie der Gammafunktion, or Courant: Differential and Integral Calculus, vol. II p. 325.

${ }^{2}$ Convexity + boundedness in some finite interval is equivalent to strong convexity. Cf. Hardy, Littlewood, Polya: Inequalities, p. 9 I.

${ }^{3}$ Konvexe Lösung der Funktionalgleichung $1 / f(x+1)=x f(x)$, Acta mathematica 70, p. 59. 
In the present paper we shall give some theorems concerning monotone or convex solutions of $(I)$ for certain general classes of functions $g(x)$. As special cases we shall obtain the theorem of Bohr, and also a somewhat weaker form of the theorem of A. E. Mayer, with "convex" replaced by "strongly convex". Also an analogous theorem for more general difference equations will be derived. Under suitable restrictions for $g(x)$ the special solutions obtained here, can be proved to be identical with the $\diamond$ Hauptlösungen» mentioned above.

Theorem A. Let $g(x)$ be defined for $x>0$, and let

$$
\text { g. 1. b. } g(x)=\text { o. }^{1}
$$

Then every two monotone non-decreasing solutions of (I) differ at most by a constant.

Proof: Let $f(x)=\varphi(x)$ and $f(x)=\psi(x)$ be two monotone non-decreasing solutions of $(1)$ for $x>0$. Then

$$
p(x)=\varphi(x)-\psi(x)
$$

is a function of period $I$ and is uniformly bounded, as the monotone functions $\varphi(x)$ and $\psi(x)$ are certainly bounded for $\mathbf{I} \leqq x \leqq 2$. Let

$$
\begin{aligned}
& M=\text { l. u. b. } p(x) \\
& m=\text { g. 1. b. } p(x) .
\end{aligned}
$$

Let $p(x)$ not be a constant. Then $M>m$. Let $\varepsilon$ be a number with $0<\varepsilon<$ $<\frac{M-m}{2}$. There is an $x_{0}>0$ such that $g\left(x_{0}\right) \leqq \varepsilon$. As $\varphi(x)$ is non-decreasing

$$
\varepsilon \geqq g\left(x_{0}\right)=\varphi\left(x_{0}+\mathrm{I}\right)-\varphi\left(x_{0}\right) \geqq \varphi(b)-\varphi(a)
$$

for all $a$ and $b$ with

$$
x_{0} \leqq a \leqq b \leqq x_{0}+\mathrm{I} .
$$

Hence, as $\psi$ is non-decreasing

$$
\varepsilon \geqq \psi(b)-\psi(\dot{a})+p(b)-p(a) \geqq p(b)-p(a)
$$

for all $a, b$ satisfying (3). Thus in particular for $b=x_{0}+\mathrm{I}$

$$
\varepsilon \geqq p\left(x_{0}+\mathrm{I}\right)-p(a)=p\left(x_{0}\right)-p(a)
$$

for all $a$ in $x_{0} \leqq a \leqq x_{0}+1$, and consequently

${ }^{1}$ g. 1. b. denotes the greatest lower bound, 1. u. b. the least upper bound. 


$$
\varepsilon \geqq \text { l. u. b. }\left(p\left(x_{0}\right)-p(a)\right)=p\left(x_{0}\right)-m .
$$

Similarly for $a=x_{0}$

$$
\varepsilon \geqq p(b)-p\left(x_{0}\right)
$$

for all $b$ in $x_{0} \leqq b \leqq x_{0}+\mathrm{I}$, and therefore

$$
\varepsilon \geqq \text { l. u. b. }\left(p(b)-p\left(x_{0}\right)\right)=M-p\left(x_{0}\right) .
$$

Adding we obtain the contradiction

$$
2 \varepsilon \geqq M-m
$$

which proves the theorem.

Theorem A does not assert the existence of a monotone solution. This is guaranteed under more restrictive conditions by

Theorem $\mathbf{A}^{\prime}$ : If $g(x)$ is non-increasing and $\lim _{x \rightarrow \infty} g(x)=0$, all non-decreasing solutions of ( 1 ) are given by

$$
f(x)=C-g(x)+\sum_{\mu=1}^{\infty}(g(\mu)-g(x+\mu))
$$

where $C$ is a constant; (in case $\sum_{\mu=1}^{\infty} g(\mu)$ converges, $f(x)$ may simply be written in the form $\left.C^{\prime}-\sum_{\mu=0}^{\infty} g(x+\mu)\right)$.

Proof: According to theorem A, it only remains to be shown, that the $f(x)$ given by (4) represents a non-decreasing solution of (I). Now the infinite series in (4) converges; for if $o<x \leqq n$, where $n$ is an integer, the expression

$$
\begin{aligned}
\sum_{\mu=1}^{N}|g(\mu)-g(x+\mu)| & \leqq \sum_{\mu=1}^{N}(g(\mu)-g(n+\mu))= \\
& =\sum_{\mu=1}^{n} g(\mu)-\sum_{\mu=1}^{n} g(N+\mu) \leqq \sum_{\mu=1}^{n} g(\mu)
\end{aligned}
$$

is bounded uniformly in $N$. Moreover it is obvious, that $f(x)$ is non-decreasing and that it is a solution of (I).

23-3932. Acta mathematica. 71. Imprimé le 4 juillet 1939. 
Example: Let $g(x)=\log \frac{x+\frac{\mathrm{I}}{2}}{x}$. Then, according to theorem $\mathbf{A}^{\prime}$

$$
\begin{aligned}
f(x) & =C-\log \frac{x+\frac{\mathrm{I}}{2}}{x}+\sum_{\mu=1}^{\infty}\left[\log \frac{\mu+\frac{\mathrm{I}}{2}}{\mu}-\log \frac{x+\mu+\frac{\mathrm{I}}{2}}{x+\mu}\right] \\
& =C+\log \left[\frac{x}{x+\frac{\mathrm{I}}{2}} \prod_{\mu=1}^{\infty}\left[\frac{\mu+\frac{\mathrm{I}}{2}}{x+\mu+\frac{\mathrm{I}}{2}} \cdot \frac{x+\mu}{\mu}\right)\right] \\
& =C+\log \left[\frac{x}{x+\frac{\mathrm{I}}{2}} \prod_{\mu=1}^{\infty} \frac{\left(\mathrm{I}+\frac{x}{\mu}\right) \sqrt{\mathrm{I}+\frac{\mathrm{I}}{\mu}}}{x+\frac{\mathrm{I}}{2}} \prod_{\mu=1}^{\infty} \frac{\mathrm{I}+\frac{\mathrm{I}}{2 \mu}}{\sqrt{\mathrm{I}+\frac{\mathrm{I}}{\mu}}}\right]
\end{aligned}
$$

Let $C^{\prime}=C+\log \prod_{\mu=1}^{\infty} \frac{I+\frac{\mathrm{I}}{2 \mu}}{\sqrt{\mathrm{I}+\frac{\mathrm{I}}{\mu}}} \cdot$ Then

$$
\begin{gathered}
f(x)=C^{\prime}+\log \left[-\frac{x}{x+\frac{\mathrm{I}}{2}} \prod_{\mu=1}^{\infty} \frac{\left(\mathrm{I}+\frac{x}{\mu}\right)\left(\mathrm{I}+\frac{\mathrm{I}}{\mu}\right)^{x+\frac{1}{2}}}{\left(\mathrm{I}+\frac{\mathrm{I}}{\mu}\right)^{x}\left(\mathrm{I}+\frac{x+1 / 2}{\mu}\right)}\right] \\
f(x)=C^{\prime}+\log \frac{\Gamma\left(x+\frac{\mathrm{I}}{2}\right)}{\Gamma(x)} .
\end{gathered}
$$

It is evident from theorem $\mathbf{A}^{\prime}$, that even every solution of

$$
f(x+\mathrm{I})-f(x)=\log \frac{x+\frac{1}{2}}{x}
$$

that is non-decreasing for sufficiently large $x$, will have to be of the form (5).

Let now $F(x)$ be a strongly convex solution of the functional equation 


$$
F(x+\mathrm{I})=\frac{1}{x \boldsymbol{F}(x)}
$$

treated by A. E. Mayer. Then $F(x)$ is either monotone non-decreasing or monotone non-increasing for sufficiently large $x$. Besides $F(x)$ is of constant sign, as $F(x)$ is continuous and according to $(7) F(x) \neq 0 . \quad F(x)$ satisfies the simpler functional equation

$$
F(x+2)=\frac{x}{x+\mathrm{I}} F(x)
$$

Therefore, if $F(x)>0$, then $F(x)$ is non-increasing for sufficiently large $x$, and if $F(x)<0, F(x)$ is non-decreasing for sufficiently large $x$. Hence $f(x)=$ $=-\log |F(2 x)|$ is a non-decreasing solution of (6) for sufficiently large $x$. Thus $F(x)$ is of the form $C \cdot \frac{\Gamma\left(\frac{x}{2}\right)}{\Gamma\left(\frac{x+\mathrm{I}}{2}\right)}$. As $F(x)$ is convex, $C>0$, and using the functional equation $(7)$, it follows that $C=\frac{\mathrm{I}}{\sqrt{2}}$.

Theorem B. Let $g(x)$ be defined for $x>0$ and let

$$
\lim _{x \rightarrow \infty} \inf \frac{g(x)}{x}=o
$$

Then every two strongly convex solutions of (I) differ at most by a constant.

Proof: If $f(x)$ is a strongly convex solution of (I), we derive from (2), replacing $\lambda$ by $\mathrm{I}-\lambda$

$$
f((\mathrm{I}-\lambda) x+\lambda y) \leqq(\mathrm{I}-\lambda) f(x)+\lambda f(y)
$$

adding this inequality to (2) it follows that

$$
f(\lambda x+(\mathrm{I}-\lambda) y)+f((\mathrm{I}-\lambda) x+\lambda y) \leqq f(x)+f(y)
$$

for $0 \leqq \lambda \leqq \mathrm{I}$. Let $a$ and $h$ be arbitrary positive numbers, then for $\lambda=\frac{a}{a+h}$, $\mathrm{I}-\lambda=\frac{h}{a+h}, y=x+a+h$ the last inequality yields

$$
f(x+h+a)-f(x+a) \geqq f(x+h)-f(x)
$$


Hence the function $u(x)=f(x+h)-f(x)$ is monotone non-decreasing for every positive $h$. Besides

$$
\mathrm{O} \leqq u(x+\mathrm{I})-u(x)=g(x+h)-g(x) .
$$

We have

$$
\text { g. l. b. }(g(x+h)-g(x))=\text { o. }
$$

For, otherwise there would exist a positive $\varepsilon$ such that

$$
g(x+h)-g(x)>\varepsilon
$$

for all $x>0$ and hence for positive integers $n$

$$
g(n h)>(n-\mathrm{I}) \varepsilon+g(h)
$$

by assumption there are arbitrarily large $x_{0}$, such that

$$
\frac{g\left(x_{0}\right)}{x_{0}}<\frac{\varepsilon}{2 h}
$$

let $n h \leqq x_{0}<(n+\mathrm{I}) h$; then,

$$
\frac{\varepsilon}{2 h}>\frac{g\left(x_{0}\right)}{x_{0}} \geqq \frac{g(n h)}{(n+\mathrm{I}) h} \geqq \frac{(n-\mathrm{I}) \varepsilon}{x_{0}}+\frac{g(h)}{x_{0}} \geqq \frac{n-\mathrm{I}}{n+\mathrm{I}} \varepsilon+\frac{g(h)}{x_{0}}
$$

whereas the expression on the right is certainly $>\frac{\varepsilon}{2} \frac{h}{h}$ for sufficiently large $x_{0}$.

According to theorem A $u(x)=f(x+h)-f(x)$ is then uniquely determined up to an additive constant $C=C_{h}$. Hence for two convex solutions $\varphi(x)$ and $\psi(x)$ of (I)

$$
p(x)=\varphi(x)-\psi(x)
$$

is a function of period $\mathrm{I}$, for which

$$
p(x+h)-p(x)=C_{h}
$$

is independent of $x$. Let again

$$
M=\text { l. u. b. } p(x), \quad m=\text { g. l. b. } p(x)
$$

and $\mathrm{o}<\varepsilon<\frac{M-m}{3}$, in case $p(x)$ is not a constant. Let $3 \geqq x_{0}>2$ be such, that $p\left(x_{0}\right) \geqq M-\varepsilon$, and let $x_{1}$ be such that $p\left(x_{1}\right) \leqq m+\varepsilon$ and $x_{0}<x_{1} \leqq 4$. 
Then for $h=x_{1}-x_{0}(\mathrm{o}<h \leqq 2)$ and any $x>0$

$$
p(x+h)-p(x)=p\left(x_{1}\right)-p\left(x_{0}\right)=C_{h} \leqq m-M+2 \varepsilon .
$$

Put $x=x_{0}-h(>0)$. Then

$$
m-M+2 \varepsilon \geqq p\left(x_{0}\right)-p\left(x_{0}-h\right) \geqq(M-\varepsilon)-M=-\varepsilon,
$$

as $M=$ l. u. b. $p(x)$. Consequently

$$
3 \varepsilon \geqq M-m
$$

which leads to a contradiction. Thus $p(x)$ is constant and theorem $\mathbf{B}$ proved.

Theorem $\mathbf{B}^{\prime}$ : Let $g(x)$ have a continuous derivative for $x>0$. Let $g^{\prime}(x)$ be monotone non-increasing and $\lim _{x \rightarrow \infty} g^{\prime}(x)=0$. Then all strongly convex solutions of (I) are of the form

$$
f(x)=C-\gamma x-g(x)-\sum_{\mu=1}^{\infty}\left(g(x+\mu)-g(\mu)-x g^{\prime}(\mu)\right)
$$

where $C=f(\mathrm{I})$ and

$$
\gamma=\lim _{n \rightarrow \infty}\left(\sum_{\mu=1}^{n} g^{\prime}(\mu)-g(n)\right)
$$

Proof: According to theorem B we only have to show, that the $f(x)$ given by (8) is a convex solution of (I). In order to prove the convergence of the infinite series, it is sufficient to note, that it obviously converges for $x=0$ and that the series $\sum_{\mu=1}^{\infty}\left[g^{\prime}(x+\mu)-g^{\prime}(\mu)\right]$, obtained by formal differentiation, converges absolutly and hence uniformly for uniformly bounded $x$ (owing to the same argument as used in the proof of theorem $\mathrm{A}^{\prime}$ ). Moreover

$$
\begin{aligned}
f(x+\mathrm{I}) & -f(x)=-\gamma-g(x+\mathrm{I})+g(x) \\
& -\sum_{\mu=1}^{\infty}\left[g(x+\mu+\mathrm{I})-g(x+\mu)-g^{\prime}(\mu)\right] \\
= & -\gamma+g(x)-\lim _{n \rightarrow \infty}\left[g(x+n+\mathrm{I})-\sum_{\mu=1}^{n} g^{\prime}(\mu)\right]=g(x),
\end{aligned}
$$


as

$$
\lim _{n \rightarrow \infty}[g(x+n+\mathrm{I})-g(n)]=\lim _{n \rightarrow \infty} \int_{0}^{x+1} g^{\prime}(n+y) d y=0
$$

besides

$$
\begin{aligned}
f(\mathrm{I}) & =C-\gamma-g(\mathrm{I})-\sum_{\mu=1}^{\infty}\left[g(\mathrm{I}+\mu)-g(\mu)-g^{\prime}(\mu)\right] \\
& =C-\gamma+\lim _{n \rightarrow \infty}\left[\sum_{\mu=1}^{n} g^{\prime}(\mu)-g(n+\mathrm{I})\right]=C .
\end{aligned}
$$

The convex character of $f(x)$ is obvious.

Note: The assumption of strong convexity of $f(x)$ cannot be weakened to convexity alone. There are under the same assumptions for $g(x)$ as in theorem $B^{\prime}$ always many other "convex" solutions, at least on the basis of Zermelo's axiom. For with $g(x)$ also $g(x)-$ I satisfies the assumptions. Thus the equation $F(x+\mathrm{I})-F(x)=g(x)$ - I has a strongly convex solution $F(x)$. Let now $\varphi(x)$ be a discontinuous solution of the functional equation $\varphi(x+y)=\varphi(x)+\varphi(y)$, which may be chosen in such a way, that $\varphi(\mathrm{I})=\mathrm{I}^{1} \quad \varphi(x)$ is convex and $\varphi(x+\mathrm{I})=\varphi(x)+\mathrm{I}$. Then $f(x)=F(x)+\varphi(x)$ is a discontinuous convex solution of our equation (I).

Example: For $g(x)=\log x$ the assumptions of theorem $\mathbf{B}^{\prime}$ are satisfied. All strongly convex solutions of (I) will then be given by

$$
f(x)=f(\mathrm{I})-\gamma x-\sum_{\mu=1}^{\infty}\left[\log \left(\mathrm{I}+\frac{x}{\mu}\right)-\frac{x}{\mu}\right]-\log x
$$

where $\gamma=\lim _{n \rightarrow \infty}\left(\sum_{\mu=1}^{n} \frac{1}{\mu}-\log n\right)$ is Euler's constant. Hence

$$
f(x)=f(\mathrm{I})+\log \Gamma(x)
$$

and we regain the theorem of $H$. Bohr.

It seems possible to extend the preceding theorems in various ways to equations of more general type than equation (I). An extension to difference equations shall be given here.

1 Such solutions are constructed by Hamel: Mathematische Annalen 6o, p. 459-462. 
Theorem C. Given the difference equation

$$
a_{0} f(x+n)+a_{1} f(x+n-\mathrm{I})+\cdots+a_{n} f(x)=g(x)
$$

with constant real coefficients $a_{k}$ for a function $f(x)$ defined for $x>0$. Let all roots of the characteristic equation

$$
F(\varrho)=a_{0} \varrho^{n}+a_{1} \varrho^{n-1}+\cdots+a_{n}=\mathrm{o}
$$

be simple and of absolute value I (real or imaginary).

If $\varrho=+$ I is a root of $(10)$, and if $\lim _{x \rightarrow \infty} g(x)=0$, then the difference of any two monotone non-decreasing solutions of (9) is a constant.

If $\varrho=+I$ is not a root of (IO) and if $\lim _{x \rightarrow \infty} \frac{g(x)}{x}=0$, then there exists at most one monotone non-decreasing solution of (9).

Proof: Let $f(x)=\varphi(x)$ and $f(x)=\psi(x)$ be two monotone non-decreasing solutions of (9). Then $\varphi(x)-\psi(x)=P(x)$ is a solution of the corresponding homogeneous equation. Hence $P(x)$ is of the form

$$
P(x)=\sum_{\mu=1}^{n} p_{\mu}(x) \varrho_{\mu}^{x},
$$

where the $p_{\mu}(x)$ are functions of period $\mathrm{I}$, determined by the system of linear equations

$$
P(x+h)=\sum_{\mu=1}^{n} p_{\mu}(x) \varrho_{\mu}^{x+h} \quad(h=0, \ldots, n-\mathrm{I}) ;^{1}
$$

the determinant of these equations is $\left(\varrho_{1} \ldots \varrho_{n}\right) x \prod_{l>k}\left(\varrho_{l}-\varrho_{k}\right) \neq 0 . \quad$ As difference of two monotone functions $P(x)$ is bounded in every positive finite interval; hence all $p_{i}(x)$ are bounded everywhere.

We cannot assert, that $P(x)$ is uniformly almost periodic in the ordinary sense, at it is not necessarily continuous. In spite of that the existence of a relatively dense set of translation numbers of $P(x)$ for every $\varepsilon>0$ is easily established. Let $\varrho_{l}=e^{i \lambda_{\tau}}$ and let $B=\operatorname{Max}\left(\mathrm{I}+\left|\lambda_{k}\right|\right)$. According to Kronecker's

${ }^{1}$ Cf. e. g. P. M. Batchelder: An introduction to linear difference equations, Chapt. I, § 4, (the proofs given there have to be modified slightly for the case of non-analytic solutions) or Nörlund 1. c. p. $295-6$. 
theorem ${ }^{1}$ there is for given $\delta>0$ a number $l$ such that every interval of length $l$ contains a solution $x$ of the $n+\mathrm{I}$ inequalities

$$
|2 \pi x| \leqq \frac{\delta}{B}, \quad\left|\lambda_{k} x\right| \leqq \frac{\delta}{B} \quad(\bmod 2 \pi) \quad(k==\mathrm{I}, \ldots, n) ;
$$

if $y$ is the integral number nearest to $x$,

$$
\left|\lambda_{k}, y\right| \leqq \frac{\delta\left(\mathbf{I}+\left|\lambda_{k}\right|\right)}{B} \leqq \delta \quad(k=\mathrm{I}, \ldots, n)
$$

Thus every interval of length $L=l+4$ contains an integral number $y$, for which all $\left|\lambda_{k} y\right| \leqq \delta(\bmod 2 \pi)$. Then

$$
\begin{aligned}
|P(x+y)-P(x)| & =\left|\sum_{l=1}^{n}\left(p_{l}(x+y) \varrho_{l}^{x+y}-p_{l}(x) \varrho_{l}^{x}\right)\right| \\
& =\left|\sum_{l=1}^{n} p_{l}(x) \varrho_{l}^{x}\left(\varrho_{l}^{y}-\mathrm{I}\right)\right| \\
& \leqq \sum_{l=1}^{n}\left|p_{l}(x)\right| \cdot\left|e^{i \delta}-\mathrm{I}\right| \leqq \varepsilon
\end{aligned}
$$

for sufficiently small $\delta=\delta(\varepsilon)$.

Let $P(x)$ not be a constant. Then there are two values $x_{0}$ and $x_{1}$, such that

$$
\left|P\left(x_{0}\right)-P\left(x_{1}\right)\right|=3 \varepsilon>0 .
$$

Hence, if $y$ is a translation number pertaining to $\varepsilon$,

$$
\left|P\left(x_{0}+y\right)-P\left(x_{1}+y\right)\right| \geqq \varepsilon
$$

Thus the total variation of $P(x)$ is $\geqq \varepsilon$ in every interval of sufficient length.

Let $N$ be such, that the total variation of $P(x)=\varphi(x)-\psi(x)$ is $\geqq \varepsilon$ in every interval of length $N$. As the total variation of $P(x)$ is at most equal to the sum of the total variations of $\varphi(x)$ and $\psi(x)$ and as those functions are monotone, we have

$$
\varphi(x+N)-\varphi(x)+\psi(x+N)-\psi(x) \geqq \varepsilon
$$

hence for every positive integer $m$

\footnotetext{
1 Cf. e. g. J. Fávard: Lecons sur les fonetions presquepériodiques, p. I8-2I.
} 


$$
\varphi(x+m N)+\psi(x+m N) \geqq m \varepsilon+\varphi(x)+\psi(x)
$$

and consequently

$$
\lim _{x \rightarrow \infty} \inf \frac{\varphi(x)+\psi(x)}{x} \geqq \varepsilon>0
$$

unless $P(x)$ is a constant.

The solutions of (9) may be found by the method of variations of constants. ${ }^{1} f(x)$ is of the form

$$
f(x)=\sum_{l=1}^{n} \varrho_{l}^{x} c_{l}(x)
$$

where $c_{l}(x)$ is a solution of the equation

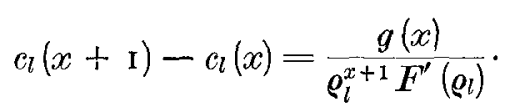

This implies

$$
c_{l}(x+k)=c_{l}(x)+\frac{\mathrm{I}}{\varrho_{l}^{x} \boldsymbol{F}^{\prime}\left(\varrho_{l}\right)} \sum_{\mu=0}^{k-1} \frac{g(x+\mu)}{\varrho_{l}^{\mu+1}} .
$$

If $\lim _{x \rightarrow \infty} g(x)=0$, we have

$$
\lim _{k \rightarrow \infty} \frac{\mathrm{I}}{k}\left|\sum_{\mu=0}^{k-1} \frac{g(x+\mu)}{\varrho_{l}^{\mu+1}}\right| \leqq \lim _{k \rightarrow \infty} \frac{\mathrm{I}}{k} \sum_{\mu=0}^{k-1}|g(x+\mu)|=0
$$

and hence

$$
\begin{aligned}
& \lim _{k \rightarrow \infty} \frac{c_{l}(x+k)}{x+k}=0, \\
& \lim _{k \rightarrow \infty} \frac{f(x+k)}{x+k}=0 .
\end{aligned}
$$

If $f(x)$ is monotone, this implies

$$
\lim _{x \rightarrow \infty} \frac{f(x)}{x}=0
$$

In particular

$$
\lim _{x \rightarrow \infty} \frac{\varphi(x)}{x}=\lim _{x \rightarrow \infty} \frac{\psi(x)}{x}=0
$$

1 Cf. Batchelder loc. cit. p. 13.

24-3932. Acta mathematica. 71. Imprimé le 4 juillet 1939. 
This constitutes a contradiction to (I 2 ) and consequently $P(x)$ is a constant. Thus the first part of theorem $C$ is proved.

If only $\lim _{x \rightarrow \infty} \frac{g(x)}{x}=0$, it follows from (I3), that at least

$$
\lim _{k \rightarrow \infty} \frac{c_{l}(x+k)}{(x+k)^{2}}=0
$$

Hence for a solution $f(x)$ of (9)

$$
\lim _{k \rightarrow \infty} \frac{f(x+k)}{(x+k)^{2}}=o
$$

and if $f$ is also monotone

$$
\lim _{x \rightarrow \infty} \frac{f(x)}{x^{2}}=0
$$

The function $\lambda(x)=\varphi(x)+\psi(x)$ is a monotone non-decreasing solution of the difference equation

$$
\sum_{\mu=0}^{n} a_{\mu} \lambda(x+\mu)=2 g(x)
$$

according to (12) and (14)

$$
\frac{\mathrm{I}}{2} \varepsilon x<\lambda(x)<x^{2}
$$

for sufficiently large $x$.

Let now $\varrho=+$ I not be a root of (Io): Then

$$
\sum_{\mu=0}^{n} a_{\mu}=\delta \neq 0
$$

Let $\sum_{\mu=0}^{n}\left|a_{\mu}\right|=A . \quad$ If

$$
\lambda(x+n)-\lambda(x)<\frac{|\delta|}{2 A}|\lambda(x)|
$$

then also

$$
\lambda(x+i)-\lambda(x)<\frac{|\delta|}{2 A}|\lambda(x)| \quad(i=0, \ldots, n)
$$

and 


$$
\begin{gathered}
\left|\sum_{\mu=0}^{n} a_{\mu} \lambda(x+\mu)-\sum_{\mu=0}^{n} a_{\mu} \lambda(x)\right|=\left|\sum_{\mu=0}^{n} a_{\mu}[\lambda(x+\mu)-\lambda(x)]\right| \\
\leqq \sum_{\mu=0}^{n}\left|a_{\mu}\right|[\lambda(x+\mu)-\lambda(x)] \leqq \frac{|\delta| \cdot|\lambda(x)|}{2}
\end{gathered}
$$

Consequently

$$
\begin{gathered}
|\delta| \cdot|\lambda(x)|-|2 g(x)| \leqq|2 g(x)-\delta \lambda(x)| \leqq \frac{|\delta| \cdot|\lambda(x)|}{2} \\
|\lambda(x)| \leqq \frac{4|g(x)|}{\delta}
\end{gathered}
$$

But for sufficiently large $x$

$$
|g(x)|<\frac{|\delta|}{8} \varepsilon \cdot x
$$

Thus $|\lambda(x)|<\frac{1}{2} \cdot \varepsilon \cdot x$, and we are led to a contradiction with (15), unless $P(x)$ is a constant or (16) does not hold.

Therefore, if $P(x)$ is not a constant, we have for all sufficiently large $x$

$$
\begin{aligned}
\lambda(x+n)-\lambda(x) & \geqq \frac{|\delta|}{2 A}|\lambda(x)|, \\
\lambda(x+n) & \geqq\left(\mathrm{I}+\frac{|\delta|}{2 A}\right) \lambda(x)
\end{aligned}
$$

as $\lambda(x)>0$ for large $x$ according to (15). But then $\lambda(x)$ would increase exponentially with $x$, which also contradicts (I 5). Hence $P(x)=$ const. $=C$. As $C$ would have to be a solution of the homogeneous equation belonging to (9), $C \cdot \delta=0$ and hence $C=0$. This completes the proof of theorem $C$.

Example: The previously considered equation

$$
f(x+\mathrm{I})+f(x)=\log x
$$

can have at most one monotone non-decreasing solution. That solution is given by

$$
f(x)=\log \frac{\sqrt{2} \Gamma\left(\frac{x+1}{2}\right)}{\Gamma\left(\frac{x}{2}\right)}
$$


Theorem D. Let $g(x)$ be defined and continuously differentiable for $x>0$, and let all roots of equation (IO) be simple and of absolute value $I$.

If $\lim _{x \rightarrow \infty} g^{\prime}(x)=0$, every two strongly convex solutions of (9) differ at most by a constant.

If + I is not a root of equation (ro) and if $\lim _{x \rightarrow \infty} \frac{g^{\prime}(x)}{x}=0$, equation (9) has at most one strongly convex solution.

Proof: If $f(x)$ is a convex solution of (9), then for $h>0 u(x)=f(x+h)-$ $-f(x)$ is a monotone non-decreasing solution of the difference equation

$$
a_{0} u(x+n)+a_{1} u(x+n-1)+\cdots+a_{n} u(x)=g(x+h)-g(x) .
$$

As $g(x+h)-g(x)=h \cdot g^{\prime}(\xi)$ we have

$$
\lim _{x \rightarrow \infty}[g(x+h)-g(x)]=0 \text { or } \lim _{x \rightarrow \infty} \frac{g(x+h)-g(x)}{x}=0
$$

respectively. Hence, according to theorem $\mathrm{C}, f(x+h)-f(x)$ is uniquely determined except for an additive constant. Let $f(x)=\varphi(x)$ and $f(x)=\psi(x)$ be two convex solutions of (9). Then $P(x)=\varphi(x)-\psi(x)$ is a solution of the homogeneous equation. Besides for $h>0$

$$
P(x+h)-P(x)=\text { const. }=C_{h}
$$

As difference of two convex functions $P(x)$ is continnous. Thus $P(x)$ is representable in the form (II) with continuous functions $p_{\mu}(x)$. The $p_{\mu}(x)$ being continuous and of period I are uniformly bounded. Therefore $P(x)$ is bounded. But from (17) $P(x+k h)=P(x)+k C_{h}$ for integers $k$. The boundedness of $P(x)$ implies, that $C_{h}=0$ for every $h>0$. Thus $\boldsymbol{P}(x)$ is a constant.

Similar uniqueness theorems may be exspected for equations of the form

$$
\int_{0}^{1} f(x+y) K(y) d y=g(x)
$$


under suitable conditions for $K$ and $g .{ }^{1}$ Of particular interest in this connection seems to be the case, in which all roots of the equation

$$
\int_{0}^{1} \rho^{y} K(y) d y=\text { o }
$$

which corresponds to the characteristic equation (10), are simple and of absolute value $I$.

${ }^{1}$ For equations of type (18) see F. John: Bestimmung einer Funktion aus ihren Integralen über gewisse Mannigfaltigkeiten, Mathematische Annalen I09, p. 488-520, and J. Delsarte: Les fonctions "moyenne-périodiques", Journal de Mathématiques pures et appliquées, vol. I00, p. 403-453. 